\title{
Retroperitoneal Hepatocellular Carcinoma Rupture Mimicking an Adrenal Hematoma
}

\author{
Chang Yeon Jung, Jung Min Bae * \\ Departments of Surgery, Yeungnam University College of Medicine, Daegu, Korea
}

\section{Article history:}

Received: December 30, 2018

Revised: October 12, 2019

Accepted: October 14, 2019

\section{*Corresponding Author:}

Jung Min Bae

Departments of Surgery, Yeungnam

University College of Medicine,

Daegu, Korea

E-mail:netetern@naver.com

\section{ORCID}

Chang Yeon Jung

https://orcid.org/0000-0002-4681-0936

Jung Min Bae

https://orcid.org/0000-0003-0923-763X

\begin{abstract}
The retroperitoneum is a posterior space of the peritoneum, which has many visceral and vascular structures. Spontaneous retroperitoneal hemorrhages have variable causes, the most common of which are diseases of retroperitoneal organs. However, retroperitoneal hemorrhages may be caused by bare area injury. In this case study, a bare area was observed in the right upper quadrant (RUQ) of the retroperitoneum, the posterosuperior region of Couinaud liver segment 7 (bare area) is directly connected to the anterior pararenal space. The rupture or exophyte of the hepatocellular carcinoma on the bare area could invade the retroperitoneum, which may lead to inaccurate diagnosis of the condition. When the mass or the hemorrhage in the RUQ of the retroperitoneum is observed in computed tomography images, it is possible that ruptured hepatocellular carcinoma or a mass on the bare area of the liver, looks like a spontaneous retroperitoneal hemorrhage in the RUQ area for example a right adrenal hemorrhage.
\end{abstract}

Keywords: adrenal hematoma, hepatocellular carcinoma rupture, spontaneous retroperitoneal hermorrahge

\section{Introduction}

The retroperitoneum is a posterior space of the peritoneum, which has many visceral and vascular structures: bowels, kidneys, urinary system, vessels, and nerves [1]. A retroperitoneal hemorrhage is a rare occurrence, with variable causes. It does not have specific symptoms, so diagnosis of a retroperitoneal hemorrhage is challenging for physicians. Spontaneous retroperitoneal hemorrhage (SRH) is defined as a retroperitoneal hemorrhage with many causes, with the exception of trauma and iatrogenic injuries [2]. SRH has non-specific symptoms [3], but the most common symptom is abdominal pain [4]. The adrenal glands are retroperitoneal organs that are shaped triangularly, on the superior aspect of each kidney [5].The right adrenal gland is near the right hemidiaphragm, liver, and inferior vena cava [5]. Some articles have reported confusion about the diagnosis for an adrenal mass observed in the right adrenal gland area or whether this was a hemorrhage [6]. This Case Study describes findings from a patient presenting with these symptoms.

\section{Case Report}

A 56-year-old male, with anticoagulant and antiplatelet medications was admitted to the emergency room, because of right upper quadrant (RUQ) pain without trauma, that persisted for 7 days. He initially visited a local medical center because he was experiencing a progressive intensity of pain which became severe. He had a non-enhanced abdominal CT scan (to determine whether there was an adrenal mass or hemorrhage), before he was transferred to the emergency room. Upon arrival his vital signs were stable (blood pressure $150 / 91 \mathrm{mmHg}$, pulse rate $88 \mathrm{bpm}$, body temperature $36.8^{\circ} \mathrm{C}$, respiratory rate $19 \mathrm{bpm}$ ), hemoglobin $11 \mathrm{~g} / \mathrm{dl}$, white blood cell count 15,410 cells $/ \mu \mathrm{L}$, platelet count was 316,000 cells $/ \mu \mathrm{L}$, and INR 1.19, and other laboratory tests were in the normal range. An enhanced abdominal CT was carried out and the 
images showed an oval shaped hemorrhage with contrast extravasation $(10.5 \times 7 \mathrm{~cm})$ on the retroperitoneum, the right kidney was dented by a collection of suprarenal area fluid, and the adrenal gland was not enhanced (Figure 1). So, the initial diagnosis was retroperitoneal hemorrhage due to adrenal gland hemorrhage because of the antiplatelet medication, or liver injury due to unconscious trauma. Due to hypertension, this patient was using an anticoagulation and an antiplatelet agent and was therefore at high risk of SRH. Use of anticoagulation and antiplatelet agents is commonly reported as a high risk
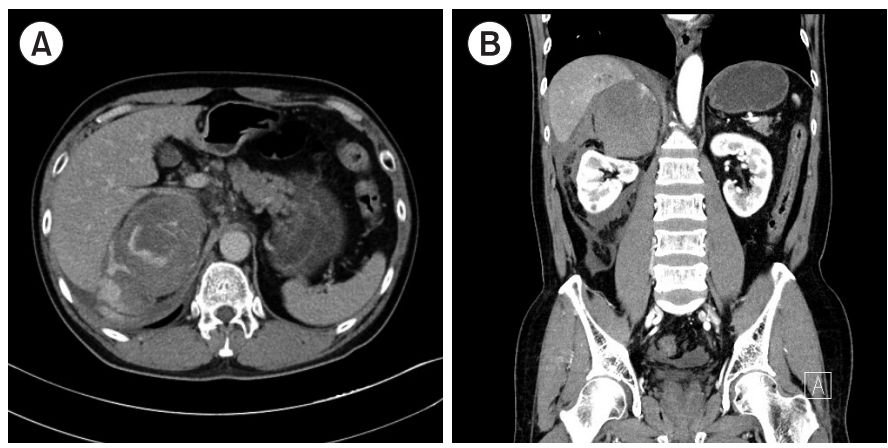

Figure 1. (A) High density lesion is on the right suprarenal area (diameter $10 \mathrm{~cm}$ ) with contrast extravasation. (B) The oval shaped hemorrhage with contrast extravasation $(10.5 \times 7 \mathrm{~cm})$ is in the right upper quadrant area of the retroperitoneum. The right kidney was dented by suprarenal area fluid collection and the adrenal gland was not enhanced.
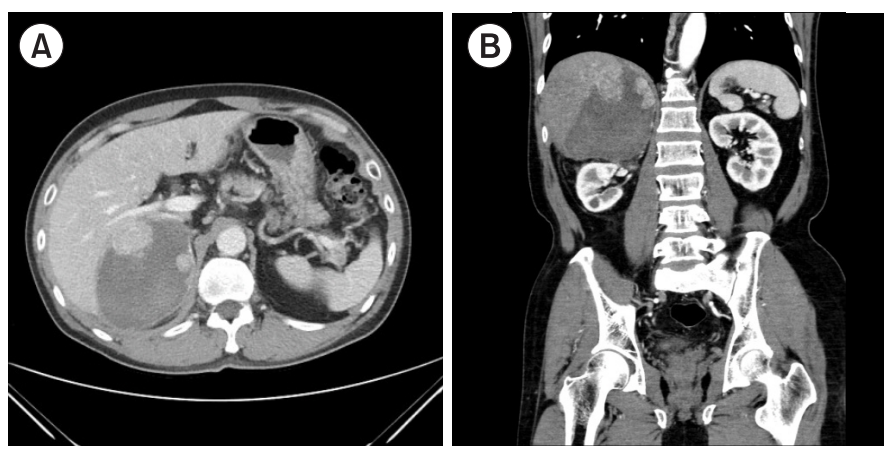

Figure 2. (A) The hematoma was $10 \times 6.8 \mathrm{~cm}$, and the mass like lesions were in the hematoma. (B) The mass like lesions were in the hematoma.
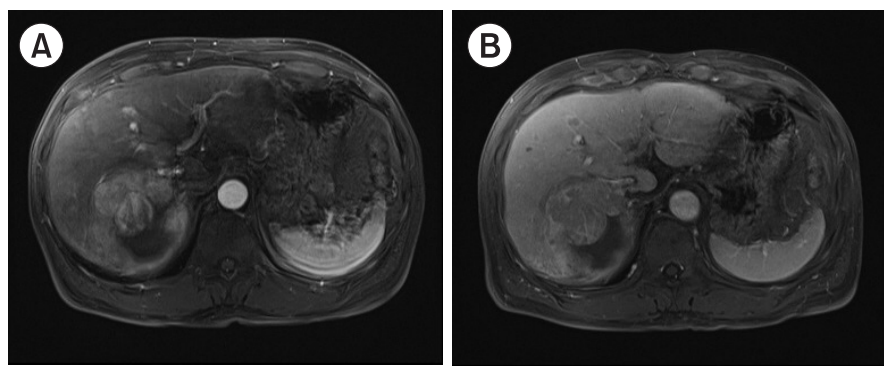

Figure 3. (A) The exophytic, polypoid masses were on segment VI, and segment VII with early enhancing in the arterial phase of the MRI scan. (B) The exophytic, polypoid masses were on segment VI and VII with washout of contrast. Many daughter nodules were in both hepatic lobes. factor for SRH but this needs to be quantified [7]. The patient was admitted to a general ward where conservative treatment [pain control, hemostatic agents (botropase, p-aminomethyl benzoic acid, tranexamic acid), and bed rest] was administered. After 8 days of hospitalization he was discharged, and 30 days following admission he had a follow up computerized tomography (CT) scan (Figure 2). On the CT scan image, the hematoma had barely been reabsorbed $(10 \times 6.8 \mathrm{~cm})$, and in Couinaud liver segment 7, an ill-defined mass was observed that was not a laceration. So, the patient had a magnetic resolution imaging (MRI) scan of his liver. The images on the MRI scan of the liver indicated exophytic, polypoid masses which showed early enhancement and delayed wash-out on Couinaud liver segment 6 and 7. Many daughter nodules were present in both hepatic lobes (Figure 3). The former CT images showed fluid collection on the retroperitoneum, and the radiological diagnosis of the patient was a retroperitoneal hemorrhage due to the rupture of a hepatocellular carcinoma in Couinaud liver segment 7. He was referred to the Department of Hepatobiliary Surgery, and received treatment including transarterial chemoembolization and chemotherapy because he had multiple hepatocellular carcinoma nodules. Transarterial chemoembolization is the first-line treatment for patients with a stable hemodynamic condition [8].

\section{Discussion}

SRH is potentially a life-threatening condition which is challenging to diagnose [4]. Many diseases such as vascular diseases, renal/adrenal tumors, renal pathology, rheumatologic diseases, and coagulopathy may cause SRH.

The RUQ area of the retroperitoneum, near the renal and adrenal area, is close to Couinaud liver segment 7 of the liver. The posterosuperior region of Couinaud liver segment 7 , also called the liver bare area, is not covered by the peritoneum, and has direct contact with the diaphragm and anterior pararenal space [9]. Due to direct contact between the bare area and perirenal space, some cases of extension of right hepatic mass have been reported as mimicking a right hepatic adrenal tumor [6]. For the same reason, the exophyte or the rupture of hepatocellular carcinoma on the bare area of Couinaud liver segment 7, could be an invasion of the retroperitoneum, and could look like an adrenal mass or hemorrhage. SRH is uncommon, but when it is encountered it is usually an emergency situation with active bleeding. So, in patients with a SRH, the blunt abdominal trauma guidelines could be useful. Focused Assessment with Sonography in Trauma (FAST) scanning is a useful tool in blunt trauma patients. For the patient who has stable vital signs, FAST scanning could identify hemoperitoneum quickly and safely. After which the CT scan is 
considered a more accurate method for identifying the origin of the bleeding [10]. However, in this case study, initial CT images yielded "dirty images," so it was not easy to distinguish sources of bleeding, and differentiate mimicry, especially in the RUQ area of the retroperitoneum where there is a space that has contact with the bare area of the liver and the suprarenal area. So, using the initial CT images, it may appear to be a RUQ area of retroperitoneal hemorrhage (it is possible that the disease originated from the bare area of the liver). If the patient had unstable vital signs, physicians may conduct an emergency operation, and precisely identify the site of bleeding or mass. However, when the patient has stable vital signs, the physicians could choose a conservative course of treatment. In this situation, if follow up CT images have not shown a remarkable decrease, or progression in size, physicians should consider a MRI scan or more diagnostic tests (e.g. for tumor markers), for more accurate diagnosis.

\section{Conflicts of Interest}

The authors have no conflicts of interest to declare.

\section{References}

[1] Manzini N, Madiba TE. The management of retroperitoneal haematoma discovered at laparotomy for trauma. Injury 2014;45(9):1378-83.

[2] Sönmez BM, Yilmaz F, Özkan FB, Ongar M, Öztürk D, Cesur F. Retroperitoneal Haematom due to Spontaneous Rupture and Haemorrhage of Adrenal Cyst Presenting with Grey Turner's Sign. J Pak Med Assoc 2015;65(7):788-9.

[3] Chan YC, Morales JP, Reidy JF, Taylor PR. Management of spontaneous and iatrogenic retroperitoneal haemorrhage: Conservative management, endovascular intervention or open surgery? Int J Clin Pract 2008;62(10): 1604-13.

[4] Sunga KL, Bellolio MF, Gilmore RM, Cabrera D. Spontaneous retroperitoneal hematoma: Etiology, characteristics, management, and outcome. J Emerg Med 2012;43(2):e157-61.

[5] Megha R, Leslie SW. Anatomy, Abdomen and Pelvis, Adrenal Glands (Suprarenal Glands). In: StatPearls. Treasure Island (FL): StatPearls Publishing; 2019.

[6] Hanai T, Miyatake R, Hashimoto K, Kato Y, Iguchi M. A case of pedunculated hepatoma suspected of adrenal tumor. Hinyokika Kiyo 1999;45(6):411-3. [in Japanese].

[7] Isokangas JM, Perala JM. Endovascular embolization of spontaneous retroperitoneal hemorrhage secondary to anticoagulant treatment. Cardiovasc Intervent Radiol 2004;27(6):607-11.

[8] Cheung TT, Poon RT, Chok KS, Chan AC, Tsang SH, Dai WC, et al. Management of spontaneously ruptured hepatocellular carcinomas in the radiofrequency ablation era. PLoS One 2014;9:e94453.

[9] Miele V, Andreoli C, De Cicco ML, Adami L, David V. Hemoretroperitoneum associated with liver bare area injuries: CT evaluation. Eur Radiol 2002;12(4):765-9.

[10] Gamanagatti S, Rangarajan K, Kumar A, Jineesh. Blunt abdominal trauma: Imaging and intervention. Curr Probl Diagn Radiol 2015;44(4):321-36. 